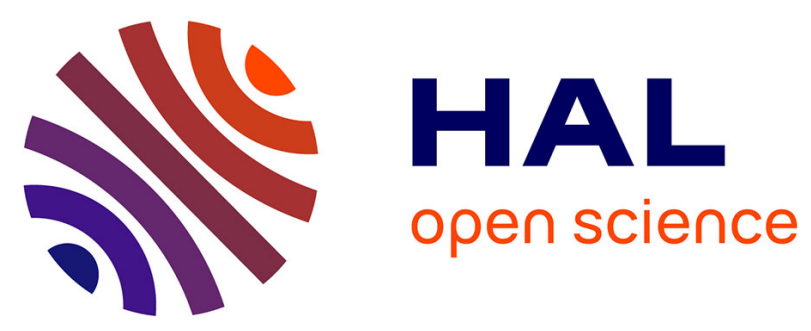

\title{
MOMAC: a SAXS/WAXS laboratory instrument dedicated to nanomaterials
}

Olivier Taché, Stéphan Rouzière, Philippe Joly, Mohamed Amara, Blaise

Fleury, Antoine Thill, Pascale Launois, Olivier Spalla, Benjamin Abécassis

\section{- To cite this version:}

Olivier Taché, Stéphan Rouzière, Philippe Joly, Mohamed Amara, Blaise Fleury, et al.. MOMAC: a SAXS/WAXS laboratory instrument dedicated to nanomaterials. Journal of Applied Crystallography, 2016, 49 (5), 10.1107/S1600576716012127 . cea-01365377

\section{HAL Id: cea-01365377 https://hal-cea.archives-ouvertes.fr/cea-01365377}

Submitted on 13 Sep 2016

HAL is a multi-disciplinary open access archive for the deposit and dissemination of scientific research documents, whether they are published or not. The documents may come from teaching and research institutions in France or abroad, or from public or private research centers.
L'archive ouverte pluridisciplinaire HAL, est destinée au dépôt et à la diffusion de documents scientifiques de niveau recherche, publiés ou non, émanant des établissements d'enseignement et de recherche français ou étrangers, des laboratoires publics ou privés. 


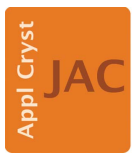

JOURNAL OF

APPLIED

CRYSTALLOGRAPHY

ISSN 1600-5767

Received 19 April 2016

Accepted 26 July 2016

Edited by G. Renaud, CEA-Grenoble DSM/ INAC/SP2M/NRS, Grenoble, France

Keywords: small-angle X-ray scattering; wideangle X-ray scattering; SAXS/WAXS; laboratory instruments; nanoparticles.

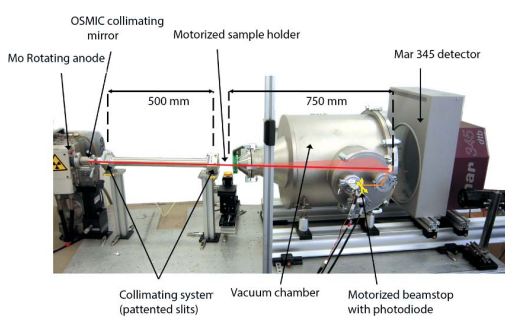

(C) 2016 International Union of Crystallography

\section{MOMAC: a SAXS/WAXS laboratory instrument dedicated to nanomaterials}

\author{
Olivier Taché, ${ }^{a}$ Stéphan Rouzière, ${ }^{\mathrm{b}}$ Philippe Joly, ${ }^{\mathrm{b}}$ Mohamed Amara, ${ }^{\mathrm{b}}$ Blaise \\ Fleury, ${ }^{a}$ Antoine Thill, ${ }^{a}$ Pascale Launois, ${ }^{b}$ Olivier Spalla ${ }^{a}$ and Benjamin Abécassis ${ }^{b} *$
}

\begin{abstract}
a'LIONS, NIMBE, CEA, CNRS, Université Paris Saclay, CEA Saclay, 91191 Gif-sur-Yvette, France, and ${ }^{\mathbf{b}}$ Laboratoire de Physique des Solides, CNRS, Université Paris-Sud, Université Paris-Saclay, 91405 Orsay Cedex, France. *Correspondence e-mail: benjamin.abecassis@gmail.com
\end{abstract}

This article presents the technical characteristics of a newly built small- and wide-angle X-ray scattering (SAXS/WAXS) apparatus dedicated to structural characterization of a wide range of nanomaterials in the powder or dispersion form. The instrument is based on a high-flux rotating anode generator with a molybdenum target, enabling the assessment of highly absorbing samples containing heavy elements. The SAXS part is composed of a collimation system including a multilayer optic and scatterless slits, a motorized sample holder, a vacuum chamber, and a two-dimensional image-plate detector. All the control command is done through a TANGO interface. Normalization and data correction yield scattering patterns at the absolute scale automatically with a $q$ range from 0.03 to $3.2 \AA^{-1}$. The WAXS part features a multilayer collimating optic and a two-dimensional image-plate detector with variable sample-todetector distances. The accessible $q$ range is $0.4-9 \AA^{-1}$, ensuring a large overlap in $q$ range between the two instruments. A few examples of applications are also presented, namely coupled SAXS/WAXS structure and symmetry determination of gold nanocrystals in solution and characterization of imogolite nanotubes and iron-filled carbon nanotube samples.

\section{Introduction}

Small- and wide-angle X-ray scattering (SAXS/WAXS) is a now well established technique whose conceptual foundations date back to 60 years ago (Guinier \& Fournet, 1955). It routinely provides quantitative structural insights on length scales ranging from 0.1 to $100 \mathrm{~nm}$. Recent developments of SAXS at synchrotron radiation facilities include access to larger length scales using collimated beams and large sampledetector distances. Furthermore, microradian diffraction exploiting refractive optics is now possible and was recently demonstrated (Petukhov et al., 2006). In the laboratory, pinhole-collimated instruments have become very popular (Pauw, 2013), and recent developments have relied on more sensitive detectors (Henrich et al., 2009) or higher flux sources such as liquid-metal jet anodes (Hemberg et al., 2003).

SAXS has been applied with great success to soft condensed matter dispersions such as surfactants, polymers, (micro-) emulsions and colloidal particles (Zemb \& Lindner, 2002). With the recent upsurge in the research effort worldwide in nanoscience and nanotechnology, the need to probe the structure of materials at nanometric length scales makes the use of SAXS highly relevant. Most of the nanomaterials being (at least partially) crystalline, WAXS techniques are also invaluable to assess order or local order at the atomic scale. Hence, a combination of SAXS and WAXS can yield a detailed structural picture of a nanomaterial at different 
length scales, from the crystallographic structure (WAXS) to the shape and size of nanoparticles in solution or on a substrate (SAXS). The organization of nanoparticles into larger superstructures such as supracrystals and their orientation under an electric or magnetic field can also be probed.

X-ray scattering has a series of advantages over other commonly used techniques for characterizing nanomaterials. First, it yields statistically relevant structural information such as the size and shape distribution or the atomic structure averaged over a very large number of objects. This precludes so-called 'cherry-picking' (the deliberate or unintentional spurious choice of a sub-population of objects in a sample) and guarantees a faithful image of the entire nanomaterial. Furthermore, the dispersion of nanoparticles can be characterized straightforwardly in solution without them being dried, as in, for example, electron microscopy. This prevents the occurrence of any artifacts prior to characterization of the sample and hence increases the reliability of the results.

In contrast to soft matter samples, classically studied using SAXS (Zemb et al., 2003), nanomaterials have specificities which make the use of $\mathrm{Cu} K \alpha(8.047 \mathrm{keV})$ radiation problematic. First, concentrated samples of nanoparticles often strongly absorb X-rays owing to their large fraction in heavy elements. Second, some important elements for nanotechnology (such as iron, for example) re-emit secondary $\mathrm{X}$-ray photons when excited with the $\mathrm{Cu} K \alpha$ wavelength, leading to a strong background and thus preventing highquality data acquisition. To circumvent these difficulties harder X-rays can be used, such as those generated by a molybdenum target (the energy of Mo $K \alpha$ is $17.4 \mathrm{keV}$ ). In this paper, we describe a custom-made state-of-the-art SAXS/ WAXS laboratory instrument, MOMAC, based on an Mo $\mathrm{X}$-ray generator and dedicated to structural investigations of nanostructured material. The design, setup and operation of the SAXS and WAXS parts of the instrument are described, as well as their calibration procedure and performance. We also present three different application examples which illustrate the relevance of this facility in the context of nanomaterial characterization.

\section{General description}

The general setup is schematically described in Fig. 1. The $\mathrm{X}$-ray generator is a molybdenum $(\lambda=0.711 \AA)$ rotating anode generator (Rigaku RUH-2R) with a point focus of $0.2 \times$ $0.2 \mathrm{~mm}$ operating at $44 \mathrm{kV}-44 \mathrm{~mA}$. Two outputs are situated on the left and on the right of the generator and deliver photons to, respectively, the WAXS and SAXS parts of the setup. In the following, we describe these two setups separately.

\section{SAXS setup}

\subsection{Optics}

A multilayer collimating mirror (Confocal Max-Flux from Osmic; Jiang et al., 2000) offers a highly monochromatic beam.
The optic uses two multilayer reflectors arranged 'side by side' to deflect X-rays in two perpendicular directions, providing two reflected beams with one reflection, and one reflected beam with two reflections which is selected. This doublebounce geometry enables high beam purity.

\subsection{Collimation system}

A first standard pair of slits placed directly after the mirror cuts the source primary beam. A second slit system is placed at $500 \mathrm{~mm}$ from the mirror and cuts the two secondary beams. This collimating system is a patented hybrid slit system (Tache \& Spalla, 2015) providing a fixed size aperture for a quick setup and a very low scattering. The beam size (FWHM) is $0.8 \times 0.8 \mathrm{~mm}$ at $500 \mathrm{~mm}$ from the optic. The flux is around $1 \times$ $10^{8} \mathrm{ph} \mathrm{s}^{-1}$.

\subsection{Sample environments}

In order to reduce the small-angle scattering signal of air, the sample zone width is only $3 \mathrm{~cm}$. An $X-Z$ motorized stage with course ranges of, respectively, 200 and $50 \mathrm{~mm}$ in the horizontal and vertical directions facilitates the positioning of the sample environments. Routinely, suspensions are put in glass or Kapton (2.1 mm internal diameter) capillaries. A three-dimensionally printed sample holder was designed to hermetically close the Kapton capillaries without glue or

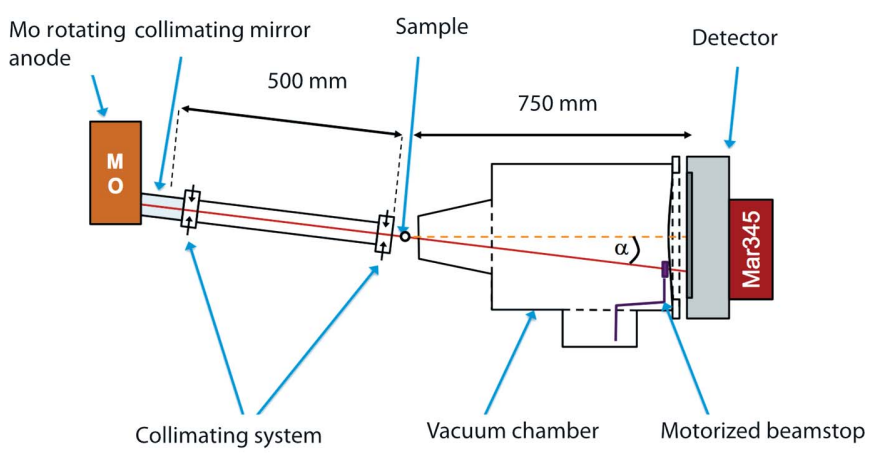

(a)

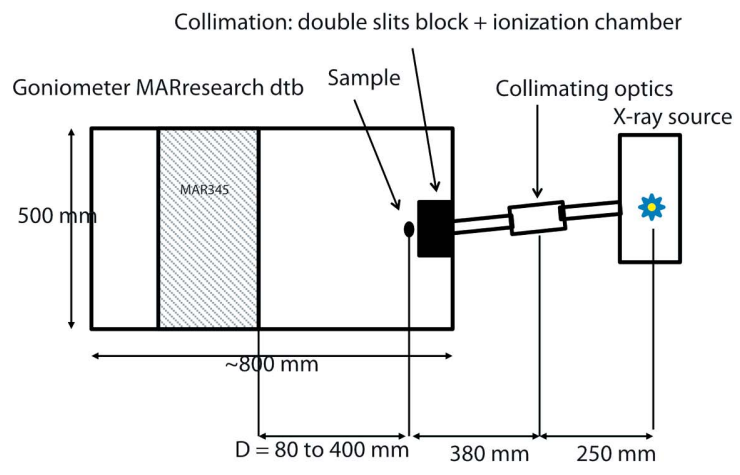

(b)

Figure 1

Schematics of the experimental setup. (a) SAXS part with the collimating mirror, sample holder, vacuum chamber and detector. (b) WAXS part with the rotating anode, the MARresearch goniometer, the collimating mirror and the detector. 


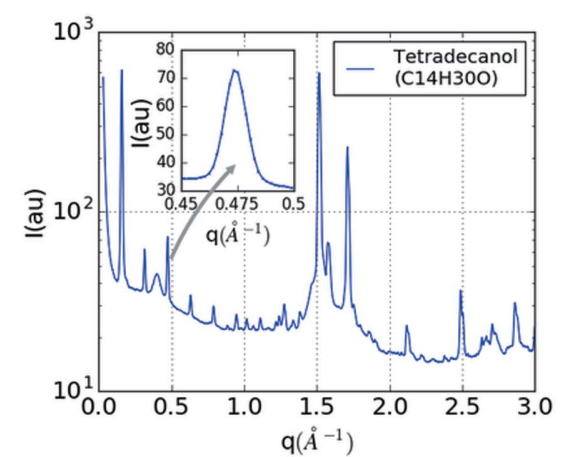

(a)

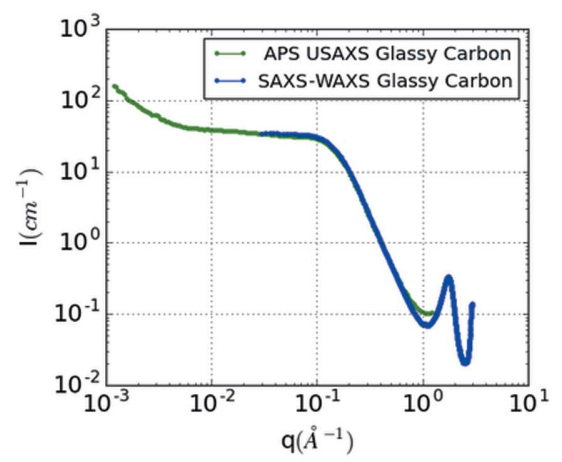

(b)

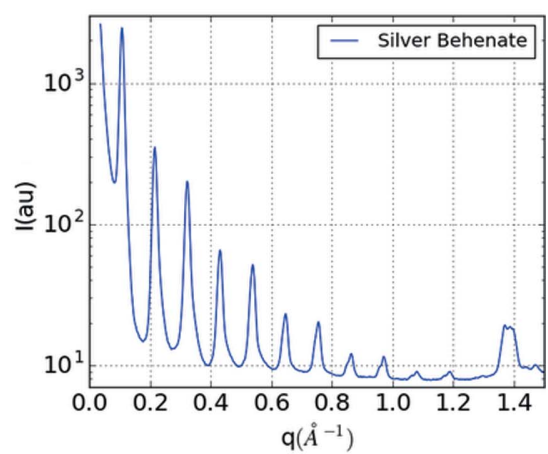

(c)

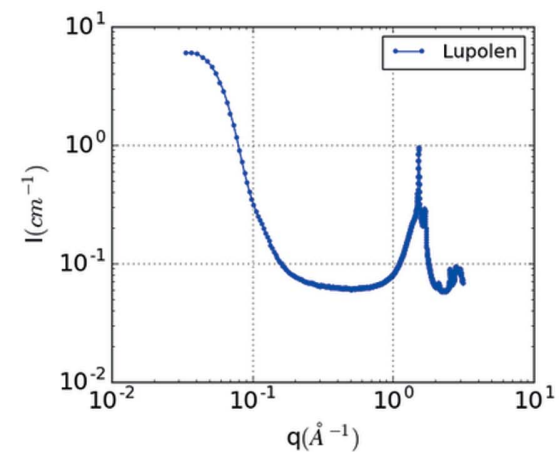

$(d)$

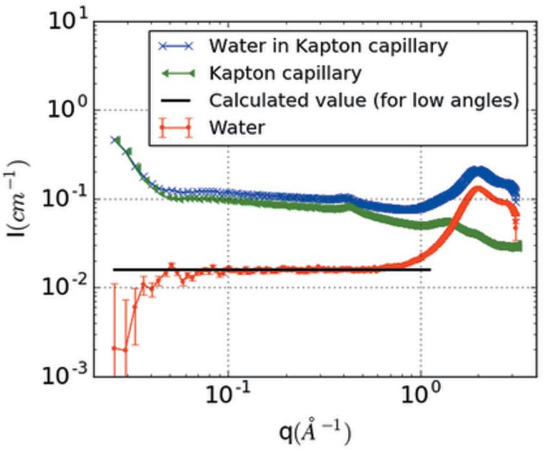

$(e)$

Figure 2

Reference samples used for calibration of the SAXS part of the instrument. (a) SAXS pattern of tetradecanol. Inset: peak used to calculate the resolution of the SAXS setup. (b) Glassy carbon, $(c)$ silver behenate, $(d)$ lupolen and $(e)$ SAXS pattern of water used as a secondary calibration sample. The different patterns in (e) correspond to the filled capillary, the empty capillary and the subtracted water diagram. The absolute value calculated for water $\left(I=1.632 \times 10^{-2} \mathrm{~cm}^{-1}\right.$ at $\left.293 \mathrm{~K}\right)$ is also plotted for comparison. For all the samples, the acquisition time was $1800 \mathrm{~s}$, except for water where it was $3600 \mathrm{~s}$.

epoxy use: we three-dimensionally printed small caps, which seal the capillaries, and a 20-position sample holder. The material used for three-dimensional printing was polylactic acid on a Makerbot Replicator2 (http://www.makerbot.com). We can also use a Linkam stage system (http://www.linkam. co.uk) for temperature-controlled experiments between 77 and $473 \mathrm{~K}$. An in-house-constructed flow-through system, using peristaltic pumps, is routinely used for kinetic studies.

\subsection{Vacuum chamber}

A $700 \mathrm{~mm}$-long vacuum chamber is placed behind the sample area. The output window has a diameter of $350 \mathrm{~mm}$ and is made of thick Mylar foil doubled by a thin aluminized foil in order to avoid ambient light penetrating inside the chamber.

\subsection{Detector}

The transmitted flux is measured with a photodiode placed on a motorized beam stop. The photodiode is a Silonex SLSD$71 \mathrm{~N} 800$ with a sensitive area of $2.7 \mathrm{~mm}^{2}$. The efficiency of the diode $\left(5.4 \mathrm{nA} \simeq 10^{8} \mathrm{ph} \mathrm{s}^{-1}\right)$ was preliminarily measured with the absolute intensities of reference samples and is consistent with the quantum efficiency calculated from the silicon thickness. The flux is measured continuously during the acquisition.
The two-dimensional detector, a MARresearch MAR345 image-plate detector with a diameter of $345 \mathrm{~mm}$ and a $100 \mu \mathrm{m}$ pixel size, is placed at a distance of $720 \mathrm{~mm}$ from the sample. The scattering vector magnitude range is from $q_{\text {min }}=0.03 \AA^{-1}$ to $q_{\max }=3.2 \AA^{-1}$. The resolution was measured by using tetradecanol (Brindley, 1981), whose diffraction peaks are very narrow. Peak shape analysis showed that the peak can be fitted with a Gaussian function with an FWHM of $0.013 \AA^{-1}$ (Fig. 2a).

\subsection{Detector geometrical setup}

We chose to turn the chamber and detector with a tilt angle $\alpha$ of around $7^{\circ}$ in order to access higher angles and hence

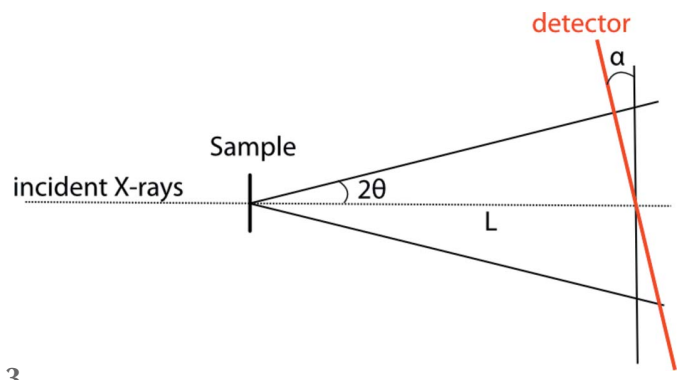

Figure 3

Scheme describing the tilt of the detector and the notation for the radial average correction. 
broaden the $q$ range. This introduces an image deformation which must be taken into account during the radial averaging process. Fig. 3 shows the scheme and the notations used for the correction.

$$
\cos (2 \theta)=\frac{L-X \cos \alpha}{\left(Y^{2}+X^{2}+L^{2}-2 L X \sin \alpha\right)^{1 / 2}},
$$

where $2 \theta$ is the Bragg angle, $L$ is the sample-detector distance where the direct beam hits the detector, $X$ and $Y$ are the coordinates of the pixel on the detector ( $X$ is in the plane of the figure, $Y$ is perpendicular) in the frame where the origin is the center of the direct beam, and $\alpha$ is the tilt angle.

\subsection{Control system}

The control system is based on the TANGO control system framework (Chaize et al., 1999). Some specific device servers were developed for the MAR345 detector and the stepper motor drivers. The user interface offers a graphic view of the experiment and a Python command line (or macros) for sequencing. The image data calibration and averaging process are done with in-house ImageJ (Schneider et al., 2012) plugins (taking the tilt angle into account) and, more recently, using the pyFAI library (Kieffer \& Karkoulis, 2013).

\subsection{Reference samples}

In order to calibrate our setup, the SAXS diagrams of three different reference samples were acquired, and these are presented in Fig. 2. The first standard (Fig. 2b) was a piece of glassy carbon obtained from Dr Jan Ilavsky (APS, Argonne National Laboratories, Chicago, USA) with the associated SAXS data acquired on the 32ID beamline at the APS, this beamline having primary calibration capability. Glassy carbon can be used as a primary standard for absolute intensity calibration thanks to its homogeneity, scattering power and resistance to radiation damage (Zhang et al., 2010). The MOMAC experimental SAXS pattern perfectly superimposes on the calibrated data. The $q$-range calibration was performed through indexing the SAXS pattern of silver behenate, which is known to display regularly spaced $(00 l)$ peaks corresponding to $d_{001}=58.380$ (3) $\AA$ spacing (Fig. 2c). Another reference sample routinely used in our laboratory is lupolen (Shaffer \& Hendricks, 1974), a $0.29 \mathrm{~cm}$-thick piece of polymer displaying constant scattering over time with a characteristic bump of absolute intensity $I=6 \mathrm{~cm}^{-1}$ at $q=0.035 \AA^{-1}$ (Fig. 2d). Finally, a Kapton capillary of known thickness $(2.1 \mathrm{~mm})$ filled with deionized water was used as a secondary absolute intensity standard (Fig. 2e). Liquid water is a preferred calibration standard for synchrotron sources, but the low scattering of this sample makes it more difficult to measure on laboratory setups (Orthaber et al., 2000). At low $q$, the SAXS pattern is perfectly flat at the expected absolute value for water $\left(I=1.632 \times 10^{-2} \mathrm{~cm}^{-1}\right.$ at $\left.293 \mathrm{~K}\right)$, showing the high quality of the instrument.

\section{WAXS setup}

\subsection{Optics}

Monochromatization is carried out by a multilayer $\mathrm{W} / \mathrm{Si}$ collimating optics (Xenocs FOX 2D 25-INF) with a divergence of $0.5^{\circ}$. The X-ray beam is further collimated by two slits to deliver an X-ray beam size of $0.8 \times 1 \mathrm{~mm}$ onto the sample. The $\mathrm{X}$-ray flux was estimated to be $10^{8} \mathrm{ph} \mathrm{s}^{-1}$ by measuring the intensity of the direct beam with the calibrated X-ray PIN diode detector provided by XENOCS. The spectral purity of the beam is higher than $97 \%$.

\subsection{Detector geometrical setup}

X-ray diffraction images are recorded on a MARresearch MARr345 image-plate detector with a diameter of $345 \mathrm{~mm}$ and a $100 / 150 \mu \mathrm{m}$ pixel size. The detector is mounted on a MARdtb goniometer, allowing one to monitor the sample-todetector distance in the range from 50 to $400 \mathrm{~mm}$, such that the accessible scattering wavevector $q$ range varies from 0.4 to $9.6 \AA^{-1}$.

\subsection{Control command and image treatment}

Detector and image acquisitions are monitored by MARresearch software.

\subsection{Reference samples}

The resolution was measured with the use of quartz and lead thiolate powders. Their diffraction peaks were fitted by using a Gaussian function with FWHM $\simeq 0.01 \AA^{-1}$. In-house software allows for angular or radial averaging of the measured intensities to obtain scattering diagrams $I(q)$ with high signal-to-noise ratio.

\section{Application examples}

In this section, we detail several applications showing the complementarity of the two parts of our experiment.

\subsection{Gold nanoparticles}

X-ray scattering from thiolated gold nanoparticles synthesized in toluene (concentration in gold of $10.5 \mathrm{mM}$ corresponding to a volume fraction of $10^{-4}$ ) (Zheng et al., 2006) was characterized using three experimental setups: a copper-based setup not described in this paper and the two instruments described here. The overlap between the three experimental $q$ ranges allowed their unification and scaling in a single diagram from 0.01 to $10 \AA^{-1}$ (Fig. 4). The X-ray signatures of both internal crystal structure and external shape and size are thus bound in a single dataset. From the SAXS part, an average size of $56.6 \pm 4.5 \AA$ was found using an analytical model for polydisperse spheres. In addition, form-factor oscillations emphasize the sample monodispersity. Gold nanoparticles being well studied, three stable structures in that size range are known with geometries Ih, Dh and TOh (Barnard et al., 2009). Under that hypothesis, the model described by Fleury et al. (2015) was applied and fitted to the data to obtain absolute 
quantification of the sample. Particles of these three symmetries were relaxed using molecular dynamics. Then, their scattering diagrams were calculated using the Debye formula. Finally, a linear combination of one TOh, one Dh and one Ih diagram, from a collection of hundreds, was selected using a fitting algorithm. It appears that the sample contains $47 \% \mathrm{Ih}$, $53 \% \mathrm{Dh}$ and $0 \% \mathrm{TOh}$.

\subsection{Imogolite nanotubes}

Imogolite nanotubes are aluminosilicate clay single-walled (SW) nanotubes having a chemical formula $(\mathrm{OH})_{3} \mathrm{Al}_{2} \mathrm{O}_{3^{-}}$ $\mathrm{Si}(\mathrm{OH})$. The aluminium atoms are in octahedral coordination and linked together through $\mathrm{Al}-\mathrm{O}-\mathrm{Al}$ bonds in a curved gibbsite-like layer. The silicon atoms have a tetrahedral coordination. They are linked to the internal surface of the aluminium dioctahedral layer with three $\mathrm{Si}-\mathrm{O}-\mathrm{Al}$ bonds. The $\mathrm{Si}-\mathrm{OH}$ groups point toward the inside of the nanotube. A technique commonly used to determine their diameter is $\mathrm{X}$-ray scattering of imogolite dry powders. In powders, the imogolite nanotubes can arrange in a variety of forms, from a Mikado-like structure of almost isolated nanotubes up to a very organized two-dimensional hexagonal packing depending upon the drying conditions. In Mikado-like structures, nanotubes have been shown to be deformed locally, adopting an ellipsoidal basis (Rouzière et al., 2016), while they adopt a hexagonal basis shape when organized in large bundles (Amara et al., 2014). Small-angle X-ray scattering on suspensions, where close contact is avoided, is a technique of choice to determine the diameter of the nanotubes. For example, $\mathrm{Al}-$ Ge imogolite with $\mathrm{Ge}$ atoms in place of $\mathrm{Si}$ atoms was first synthesized in 1982 (Wada \& Wada, 1982) and was considered to have the same shape as natural imogolite. Using SAXS on Al-Ge imogolite suspensions, Maillet et al. (2010) discovered

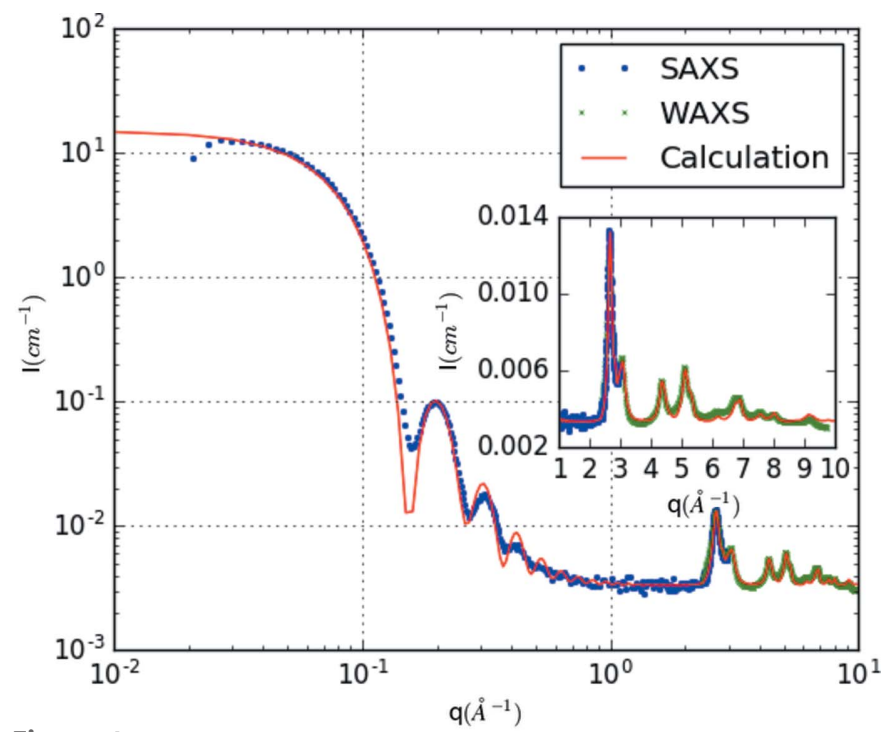

Figure 4

SAXS/WAXS unified diagram of gold nanoparticles (blue and green dots) and the corresponding fit (red line) using a linear combination of scattering diagrams of Ih-6525 (47\%) and Dh-5529 (53\%) gold nanoparticles relaxed using molecular dynamics. that they are in fact single walled or double walled depending on the synthesis conditions and proposed mean diameters. With the SAXS setup of the MOMAC apparatus, the extension of the available $q$ range toward larger scattering vectors allows one to obtain additional information on the imogolite wall structure. The scattering diagram measured on an SW AlGe imogolite is shown in Fig. 5. The pink line represents a fit to the minima in scattered intensity for the theoretical factor of an infinite homogeneous cylinder with an external radius of $2.03 \mathrm{~nm}$ and a wall thickness of $0.65 \mathrm{~nm}$. At large scattering angles, an asymmetric peak, around $1.5 \AA^{-1}$, related to the periodicity of the structure along the tube axis, is clearly observed. The scattering diagram above $1 \AA^{-1}$ thus provides valuable information on the atomic structure of the nanotubes in suspension, which was not available on standard SAXS diffractometers (Amara, 2014). The combination of the SAXS and WAXS part of MOMAC is thus a most interesting tool to study the imogolite structure.

Interestingly, single-walled nanotubes of the imogolite analog $(\mathrm{OH})_{3} \mathrm{Al}_{2} \mathrm{O}_{3} \mathrm{Ge}(\mathrm{OH})$ can be assembled into a fiber thanks to a lyophilization process. The fiber is set horizontally, perpendicular to the X-ray beam, on a goniometer head on the WAXS part of the MOMAC instrument. Nanotubes are preferentially aligned along the fiber axis and they are periodic along their axis (period $T \simeq 0.85 \mathrm{~nm}$ determined from the $00 l$ positions). The obtained WAXS pattern in Fig. 6 is typical of a fiber of aligned periodic one-dimensional objects (Vainshtein, 1966). Diffuse scattering located in (00l) planes $\left(q_{z}=2 \pi l / T\right)$ leads, when projecting their intersections with the Ewald sphere onto the planar detector, to hyperbolas in

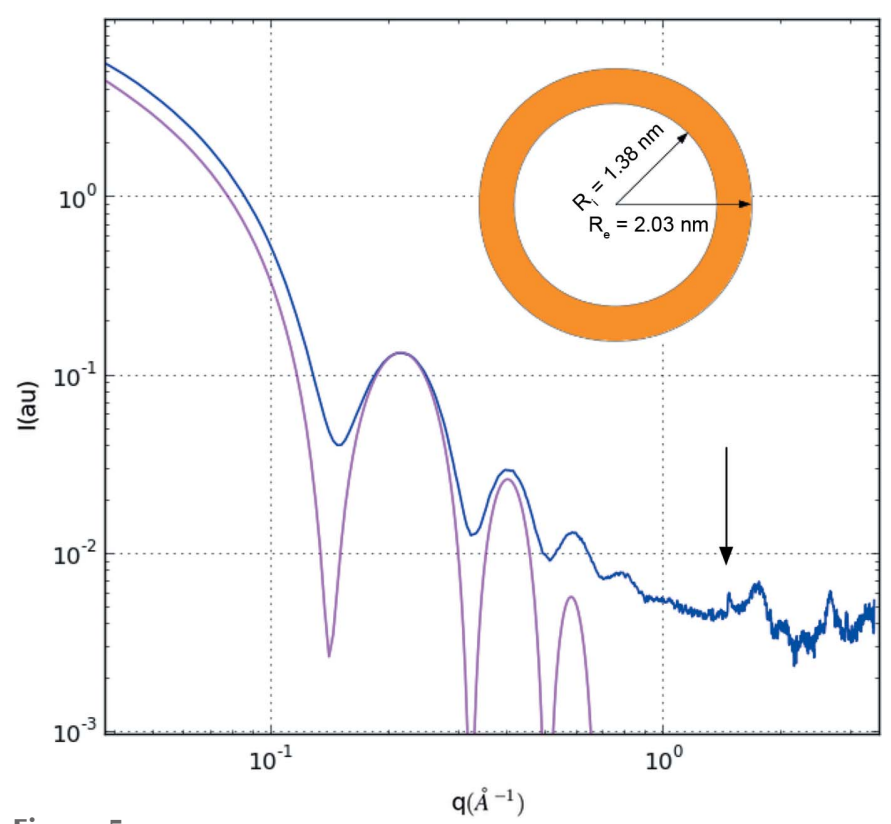

Figure 5

X-ray scattering pattern of a dispersion of single-wall $(\mathrm{OH})_{3} \mathrm{Al}_{2}$ $\mathrm{O}_{3} \mathrm{Ge}(\mathrm{OH})$ imogolite in water. The diagram was acquired using calibrated Kapton capillaries and the water signal was subtracted. The black arrow points towards the asymmetric peak linked to the periodicity of the tubes. Note the weakness of the signal at high $q$, which requires precise normalization. The pink curve is a fit to a model of a monodisperse tube with the dimensions indicated in the scheme. 


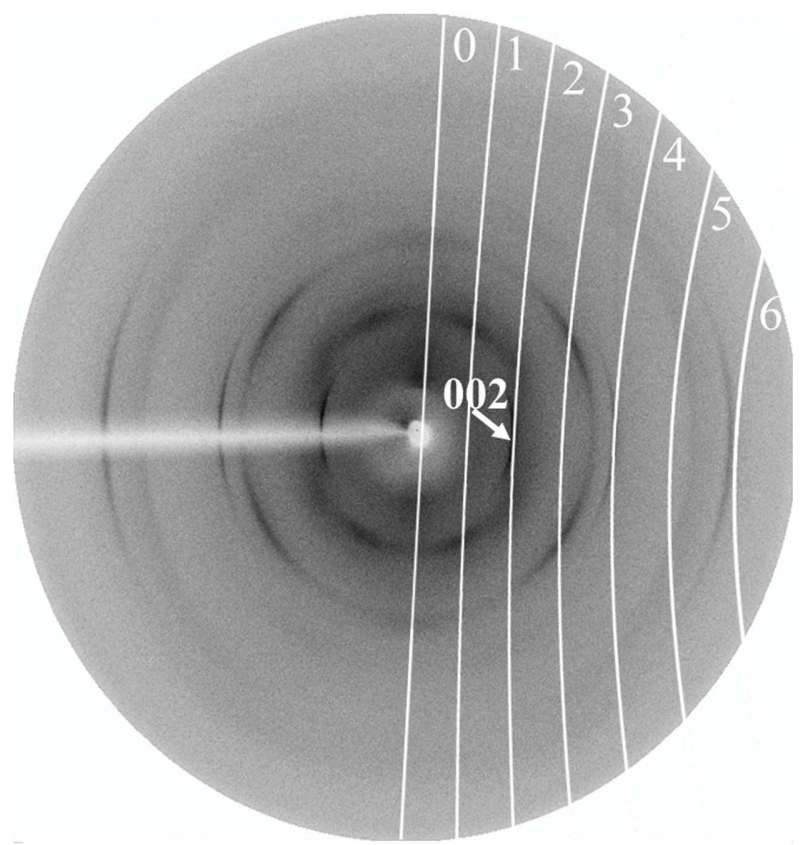

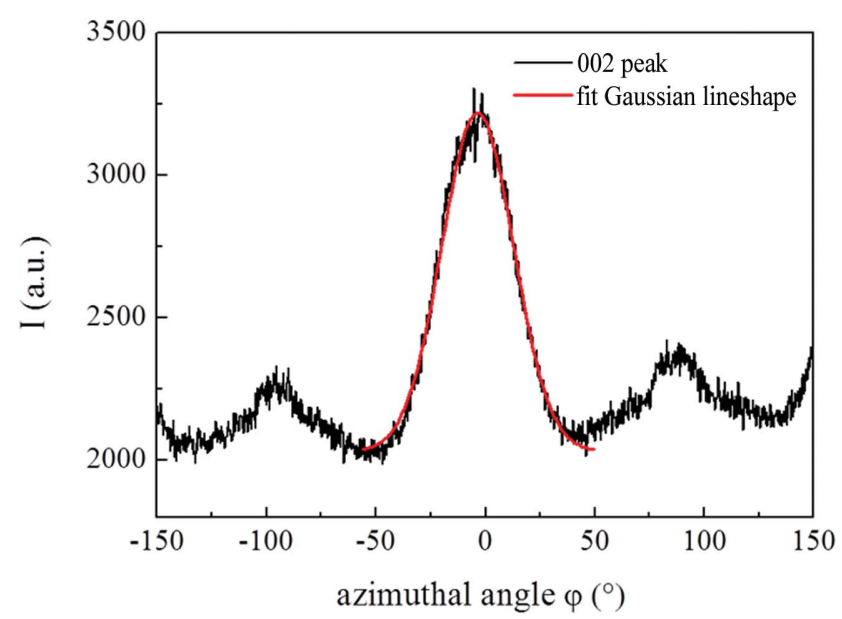

(b)

Figure 6

$(a)$

(a) WAXS pattern of SW imogolite fiber. The main $(00 l)$ reflections are indicated. White dashed lines are eye-guides for $l$ layers. The fiber axis is set horizontal, the sample-to-detector distance is $250 \mathrm{~mm}$ and the exposure time is $10 \mathrm{~h}$. The maximum accessible scattering wavevector value on the pattern is $5 \AA^{-1}$. (b) Measured intensity along the circle crossing the 002 peak as a function of the azimuthal angle $\varphi$. The best fit is obtained with a Gaussian lineshape with an FWHM $=38.6^{\circ}$.

the pattern. Measurement of the azimuthal intensity distribution in Fig. 6(b) allows one to quantify nanotube alignment with respect to the fiber axis (Pichot et al., 2006). In brief, the obtained WAXS two-dimensional pattern probes both the crystallinity of the nanotubes and their degree of alignment inside the fiber.

\subsection{Iron-filled carbon nanotubes}

Carpets of vertically aligned multiwalled carbon nanotubes (VA-MWCNT) can be synthesized in large amounts by aerosol-assisted catalytic chemical vapor deposition (Pinault et al., 2005). The samples also contain different iron-based nanocrystals or nanowires, such as iron oxide $\mathrm{Fe}_{3} \mathrm{O}_{4}$, cementite $\mathrm{Fe}_{3} \mathrm{C}$, body-centered-cubic ferrite $\alpha$-Fe and face-centeredcubic austenite $\gamma$-Fe, which are mainly located inside the nanotubes (Heresanu et al., 2008; Cambedouzou et al., 2013). The present sample was synthesized during an in situ timeresolved X-ray diffraction study of carpet growth in a synchrotron experiment (Landois et al., 2011, 2015) where the carpet was grown on a $10 \mu \mathrm{m}$-thick silicon wafer to reach a final height of $120 \mu \mathrm{m}$. It was demonstrated that $\mathrm{Fe}_{3} \mathrm{C}$ crystalline nanoparticles are the nucleation seeds for CNT growth and that $\gamma$-Fe nanowires are formed inside the carbon nanotubes at the high-temperature synthesis stage (Landois et al., 2015), while $\alpha$-Fe and $\mathrm{Fe}_{3} \mathrm{O}_{4}$ crystalline phases appear during the cooling to ambient temperature. Further ex situ experiments have been performed on a WAXS experimental setup where the VA-MWCNT carpet is placed in transmission geometry, in such a way that the normal to the substrate is perpendicular to the X-ray incident beam. They provide information about the nanotube alignment as well as about preferred orientations of iron-based nanoparticles and nanowires inside the nanotubes (Pichot et al., 2004). The main advantages of the experimental setup are (i) to give access to a wide range of scattering wavevectors characteristic of both VA-MWCNT and iron-based nanoparticles, and (ii) to allow, thanks to the two-dimensional detector, for the measurement of the whole anisotropic scattering pattern of VA-MWCNT carpets. In Fig. 7(a), the different iron-based crystalline phases $\left(\alpha-\mathrm{Fe}, \gamma-\mathrm{Fe}, \mathrm{Fe}_{3} \mathrm{C}\right.$ and $\left.\mathrm{Fe}_{3} \mathrm{O}_{4}\right)$ are identified along with the MWCNT characteristic diffraction rings. The preferential crystalline orientation of $\gamma$-Fe nanowires within the nanotube cores has been determined using an in-house computer program developed at Laboratoire de Physique des Solides. It allows one to calculate, taking into account the geometry of the experiment, a scattering image that can be compared directly with the measured one, as well as scattering diagrams to be compared with those deduced from the measured twodimensional image (see Fig. 7b). Our input parameters are the distribution of orientations of the nanotubes with respect to the normal of the carpet and, for iron-based phases, the [hkl] crystallographic axes of the iron phase along the tube. The orientation distribution function of MWCNT is deduced from the angular distribution intensity along the 002 diffraction ring at $q=1.83 \AA^{-1}$, which corresponds to the mean distance of $\sim 0.34 \mathrm{~nm}$ between concentric graphene sheets of MWCNT (Pichot et al., 2006). Note that, for a nanotube powder, the intensity would be constant. The distribution of orientation with respect to the normal of the carpet basis is characterized 


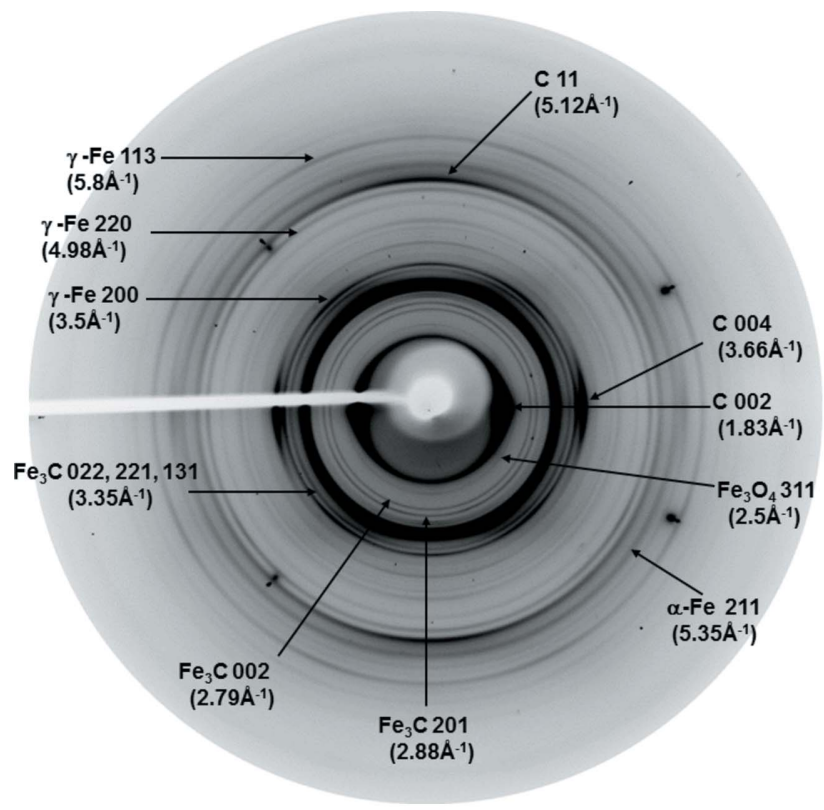

(a)

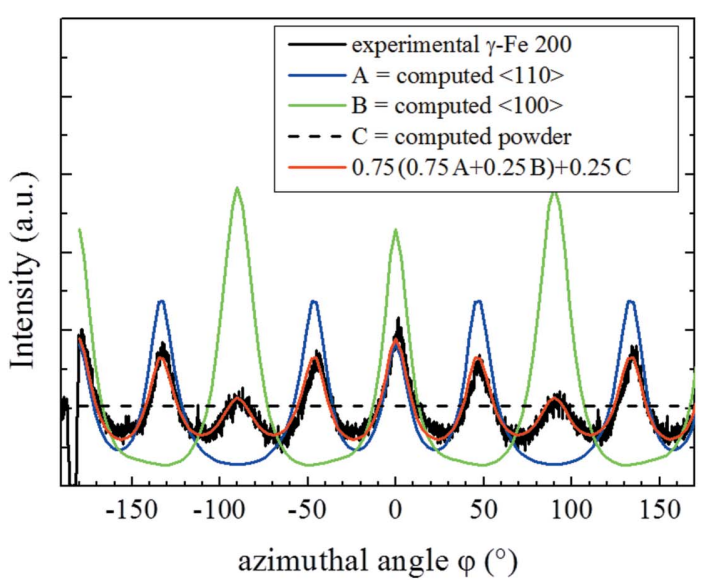

(b)

Figure 7

(a) WAXS pattern of a VA-MWCNT carpet. All scattering rings are indexed according to MWCNT and iron-based phases, as is illustrated for several of them in the figure, where their scattering wavevectors are also indicated. The four localized Bragg peaks originate from the Si wafer. The exposure time is $4 \mathrm{~h}$ and the sample-to-detector distance is $150 \mathrm{~mm}$. (b) Measured intensity along the circle at $q \simeq 3.5 \AA^{-1}$ (reflection 200 of $\gamma$-Fe) as a function of the azimuthal angle $\varphi$. The black solid line represents experimental data, solid blue and green lines represent calculations for the [110] and [100] axes of $\gamma$-Fe along the tubes, and the dashed black line corresponds to random orientation. The red line represents the best agreement for the sum of the different contributions, which fits the experimental curve.

by a Lorentzian to the power 1.5 as a function of the angle between the normal of the carpet and the nanotube long axis, with an FWHM $2 w$. The best fit of the modulation is obtained with $w=6^{\circ}$. The scattering pattern of the $\gamma$-Fe phase has been simulated considering different $[\mathrm{hkl}]$ axes along the tubes, for the orientation distribution determined for the CNT. In Fig. $7(b)$, we show that the intensity modulations along the (200) diffraction ring of $\gamma$-Fe, at $q=3.5 \AA^{-1}$, are well fitted taking into account two different crystallographic orientations of the nanowires and a proportion of nanowires with random orientation. About $25 \%$ of $\gamma$-Fe nanowires exhibit random orientations, and the remaining $75 \%$ exhibit two preferential orientations with $75 \%$ of them having [110] orientation and $25 \%$ [100] orientation.

\section{Conclusion}

A new SAXS/WAXS laboratory instrument dedicated to the study of nanomaterials has been designed and constructed. A molybdenum rotating anode is used as high-flux source for two complimentary experiments spanning a broad range of scattering vectors. We have detailed several application examples, such as gold colloidal nanocrystals and iron-filled carbon nanotubes, but other systems have also been investigated. Numerous users from various backgrounds have used the instrument seamlessly thanks to its ergonomics and high degree of automation. Future prospects include the development of other sample environments (high- and low-tempera- ture measurements, electric and magnetic fields, sample vacuum chamber).

\section{Acknowledgements}

J. Cambedouzou (ICSM/ENSM, Marcoules) is acknowledged for scientific collaboration at the beginning of the project and P. Haltebourg for mechanics. The instrument has been funded in large part by the RTRA 'triangle de la physique'. PL and SR thank the French National Agency of Research for funding the collaborative project (ANR-ALUCINAN project, reference: ANR-07-NANO-014) whereby the CNT carpet was synthesized, and Martine Mayne-L'Hermite (Laboratoire Francis Perrin, NIMBE/IRAMIS/CEA), Mathieu Pinault (Laboratoire Francis Perrin, NIMBE/IRAMIS/CEA) and P. Landois (Laboratoire Charles Coulomb, Montpellier) for our fruitful collaboration on in situ study of SWCNT carpets.

\section{References}

Amara, M. (2014). PhD thesis, Université Paris-Sud, France.

Amara, M. S., Rouzière, S., Paineau, E., Bacia-Verloop, M., Thill, A. \& Launois, P. (2014). J. Phys. Chem. C, 118, 9299-9306.

Barnard, A. S., Young, N. P., Kirkland, A. I., van Huis, M. A. \& Xu, H. (2009). ACS Nano, 3, 1431-1436.

Brindley, G. (1981). Clays Clay Miner. 29, 67-68.

Cambedouzou, J., Landois, P., Rouzière, S., Pinault, M., Mocuta, C., Hennet, L., Thiaudière, D., Mayne-L'Hermite, M. \& Launois, P. (2013). Phys. Rev. B, 88, 081402. 
Chaize, J. M., Götz, A., Klotz, W. D., Meyer, J., Perez, M. \& Taurel, E. (1999). Proceedings of ICALEPCS'99, pp. 475-479. Elettra Sincrotrone Trieste

Fleury, B., Cortes-Huerto, R., Taché, O., Testard, F., Menguy, N. \& Spalla, O. (2015). Nano Lett. 15, 6088-6094.

Guinier, A. \& Fournet, G. (1955). Small-Angle Scattering of X-rays. New York: Wiley.

Hemberg, O., Otendal, M. \& Hertz, H. (2003). Appl. Phys. Lett. 83, 1483-1485.

Henrich, B., Bergamaschi, A., Broennimann, C., Dinapoli, R., Eikenberry, E., Johnson, I., Kobas, M., Kraft, P., Mozzanica, A. \& Schmitt, B. (2009). Nucl. Instrum. Methods Phys. Res. Sect. A, 607, 247-249.

Heresanu, V., Castro, C., Cambedouzou, J., Pinault, M., Stephan, O., Reynaud, C., Mayne-L'Hermite, M. \& Launois, P. (2008). J. Phys. Chem. C, 112, 7371-7378.

Jiang, L., Verman, B. \& Joensen, K. D. (2000). J. Appl. Cryst. 33, 801803.

Kieffer, J. \& Karkoulis, D. (2013). J. Phys. Conf. Ser. 425, 202012.

Landois, P., Pinault, M., Rouzière, S., Porterat, D., Mocuta, C., Elkaim, E., Mayne-L'Hermite, M. \& Launois, P. (2015). Carbon, 87, 246-256.

Landois, P., Rouzière, S., Pinault, M., Porterat, D., Mocuta, C., Elkaim, E., Mayne-L'Hermite, M. \& Launois, P. (2011). Phys. Status Solidi B, 248, 2449-2453.

Maillet, P., Levard, C., Larquet, E., Mariet, C., Spalla, O., Menguy, N., Masion, A., Doelsch, E., Rose, J. \& Thill, A. (2010). J. Am. Chem. Soc. 132, 1208-1209.

Orthaber, D., Bergmann, A. \& Glatter, O. (2000). J. Appl. Cryst. 33, 218-225.
Pauw, B. R. (2013). J. Phys. Conden. Matter, 25, 383201.

Petukhov, A. V., Thijssen, J. H. J., 't Hart, D. C., Imhof, A., van Blaaderen, A., Dolbnya, I. P., Snigirev, A., Moussaïd, A. \& Snigireva, I. (2006). J. Appl. Cryst. 39, 137-144.

Pichot, V., Badaire, S., Albouy, P., Zakri, C., Poulin, P. \& Launois, P. (2006). Phys. Rev. B, 74, 245416.

Pichot, V., Launois, P., Pinault, M., Mayne-L'Hermite, M. \& Reynaud, C. (2004). Appl. Phys. Lett. 88, 473-475.

Pinault, M., Pichot, V., Khodja, H., Launois, P., Reynaud, C. \& Mayne, M. (2005). Nano Lett. 5, 2212-2215.

Rouzière, S., Amara, M. S., Paineau, E. \& Launois, P. (2016). Nanosized Tubular Clay Minerals, Halloysite and Imogolite, Developments in Clay Science, Vol. 7, edited by P. Yuan, A. Thill \& F. Bergaya, ch. 11. Amsterdam: Elsevier.

Schneider, C. A., Rasband, W. S. \& Eliceiri, K. W. (2012). Nat. Methods, 9, 671-675.

Shaffer, L. B. \& Hendricks, R. W. (1974). J. Appl. Cryst. 7, 159-163.

Tache, O. \& Spalla, O. (2015). WO 201 1/135510 Al. US Patent 9153351.

Vainshtein, B. (1966). Diffraction of X-rays by Chain Molecules. Amsterdam, London, New York: Elsevier Publishing Company.

Wada, S.-I. \& Wada, K. (1982). Clays Clay Miner. 30, 123-128.

Zemb, T. \& Lindner, P. (2002). Neutrons, X-rays and Light: Scattering Methods Applied to Soft Condensed Matter. Amsterdam: Elsevier.

Zemb, T., Tache, O., Ne, F. \& Spalla, O. (2003). Rev. Sci. Instrum. 74, 2456-2462.

Zhang, F., Ilavsky, J., Long, G. G., Quintana, J. P. G., Allen, A. J. \& Jemian, P. R. (2010). Metall. Mater. Trans. A, 41, 1151-1158.

Zheng, N., Fan, J. \& Stucky, G. D. (2006). J. Am. Chem. Soc. 128, $6550-6551$. 